\title{
Animated Software Training Via the Internet: Lessons Learned
}

\author{
Carol J. Scott, \\ Jet Propulsion Laboratory, California Institute of Technology \\ 4800 Oak Grove Drive M/S 301-270 \\ Pasadena, California, USA 91109-8099 \\ (818) 393-0551 \\ carol.j.scott@jpl.nasa.gov
}

\begin{abstract}
The Mission Execution and Automation Section, Information Technologies and Software Systems Division at the Jet Propulsion Laboratory, recently delivered an animated software training module to train operators at the Deep Space Network for the TMOD UPLINK Consolidation Task. The animated training software was presented as a Command Subsystem simulation for on-line instruction. Animation was developed as a prototype (papers [1][2] describing the initial development of this animation were presented at earlier conferences) and became a design and training tool. From development through delivery, significant animation design changes were made to keep pace with the changing Command formats and techniques being developed for the Deep Space Network, thus enabling training software to remain concurrent with subsystem development.
\end{abstract}

\section{TABLE OF CONTENTS}

\section{INTRODUCTION \\ 2. CHANGES IN LOOK AND FEEL \\ 3. Changes In CODE STRUCTURE \\ 3. Changes In TRAINING SCENARIOS \\ 4. DELIVERY BONUS \\ 5. CONCLUSIONS}

\section{INTRODUCTION}

The original objective was to construct a prototype for webbased delivery of mission software training that provided a mechanism for operators and engineers to master unique, operations-specific software subsystems. This training delivery had to accommodate operator and operations schedules, without interfering with hardware or software delivery schedules. The resulting training module was a web-deliverable software simulation tool available on demand for initial training, operator practice, and peer review of operating procedures.

Initial exposure for the user population occurred during the development of the animated training package with feedback used both for training modification and for system enhancements. The training prototype evolved into a software simulation utilizing the capabilities of the vector animation development software (Macromedia's Flash4). An animated simulation remained on-line, in a user-ready format, during the development cycle. Frequent access (measured hits on the site) indicated significant early interest in the tool, and provided an avenue for user feedback to incorporate enhancements into the design. The formal introduction was in the form of a demonstration to the target operations audience; it presented a mature development that had evolved dynamically thanks to inputs from developers and system engineers.

\section{CHANGES In LOOK AND FEEL}

The look and feel of the delivered training animation differed significantly from the original design due to continuing enhancements for optimizing on-line delivery. Figure 1 depicts the original design. Shortcomings of this design included color scheme, clumsy buttons, limited options, and lack of instructional information. Too many clicks and page changes were required to get smooth information flow. There was no convenient spot to place shortcuts that could be maintained in the same location on all scenes of the animation. The original version did not include simulation scenarios for operational GUIs, instead, it presumed that transactional information would be sequentially described based on operator inputs to the simulation, rather than enacted as plausible output based on inputs.

Color became a problem when system developers began providing screen dumps that differed in appearance from their original images. They used personalized palates

${ }^{1}$ U.S. Government work not protected by U.S. copyright 


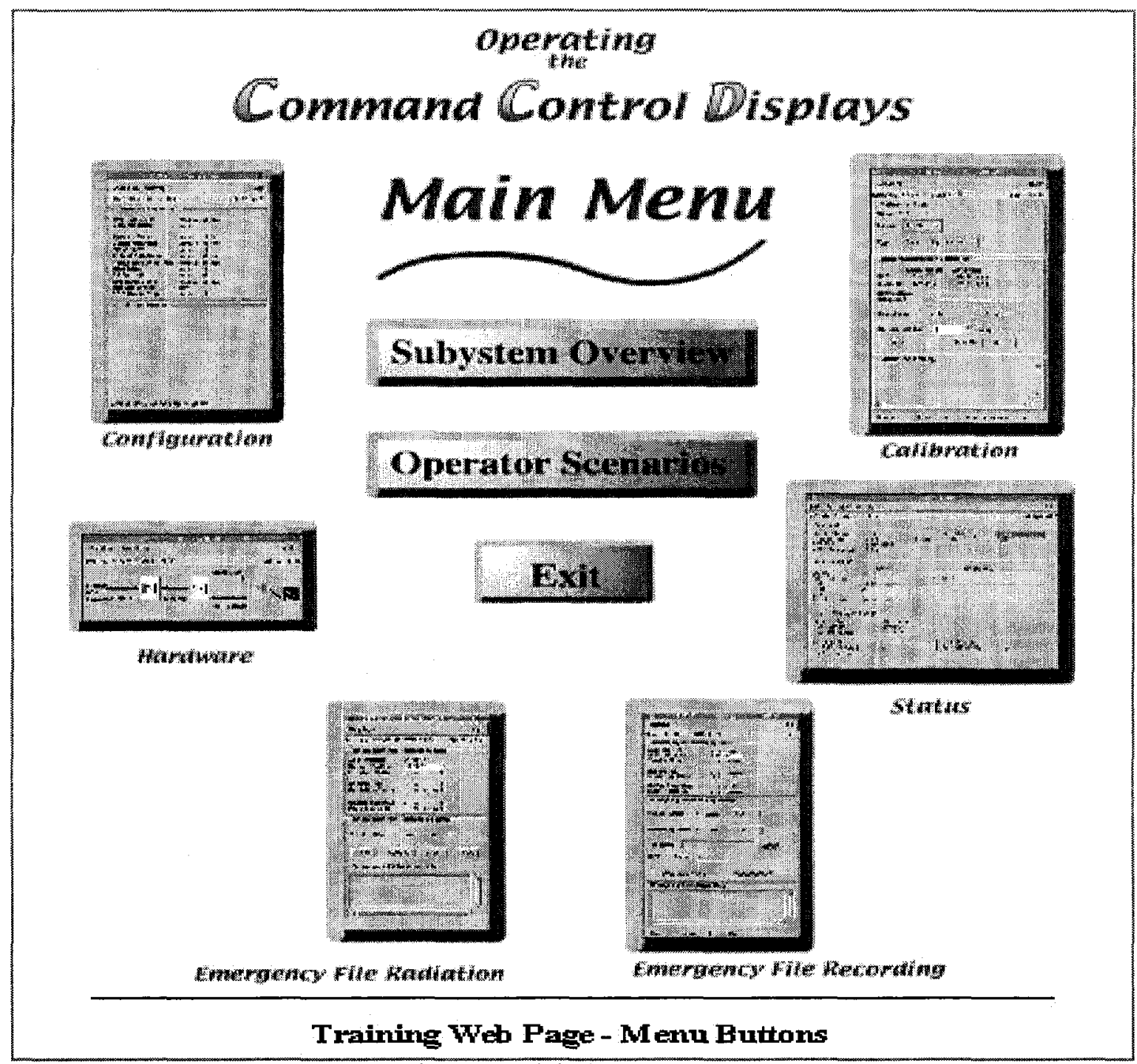

Figure 1: Original Appearance

during their login sessions, which produced graphical user interfaces (GUIs) that differed from their original color schemes. By using a multi-colored format, the animation could support most any color GUI.

Buttons were streamlined to enable greater functional access. Invisible buttons were placed over representative pictures to help identify functions (i.e. Q\&A for "questions and answers".) A row of buttons accompanied the list of menu options for getting started, and the individual GUI pictures were overlaid with invisible buttons to enable immediate access.

The original version limited navigational options from the Main Menu into the training course. By supplying the delivered version with additional labeled categories plus a button bar with fixed icon buttons common to all scenes, the operator had a common and dependable link to any point in the animation. The contents of the button bar could vary from scene to scene to avoid confusion as to which icons were actually usable in a given scene, while the positioning of the icons remained constant.

Fixed overlay display information in the original version was often an irritant to the more experienced. The delivered version provided information in the form of rollover popups that could be enabled/disabled by the operator as required. This reduced the clicking effort and the information flowed smoothly when needed.

The actual simulation capabilities were incorporated as it became apparent that the software could efficiently reproduce the desired responses to real-time inputs. The outputs of course are simulated, and all calculated outputs are fictionally derived. However, they appear in the proper sequence and represent results in the proper format and location as the operator might expect from the actual subsystem. 


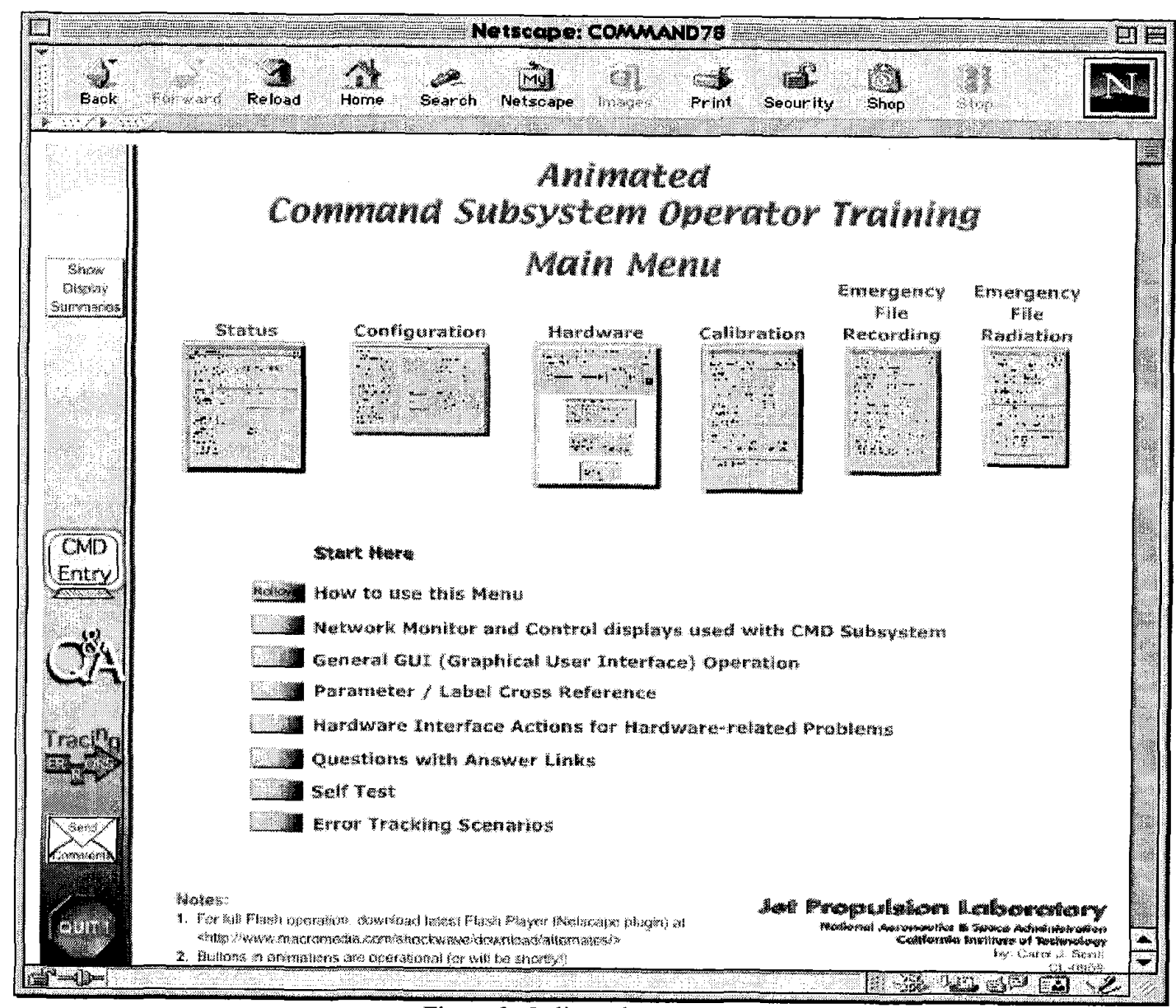

Figure 2: Delivered Appearance

Figure 2 depicts the delivered version of the animation. This version is more flexible than the original version, providing information without screen changes. The words "Start Here" direct the user where to start. By rolling over buttons while pop-ups are enabled, descriptive pop-ups appear that describe the function or capability selected. For example, Figure 3 depicts a pop-up describing the button capabilities of the Main Menu, including a small image of each of the Button Bar buttons and information on how they are used.

The pop-up enable/disable button is bright yellow on the Button Bar, and when enabled (clicked), simply rolling over one of the GUI buttons will produce the related instructional description of the capabilities of that GUI (see Figure 4.)

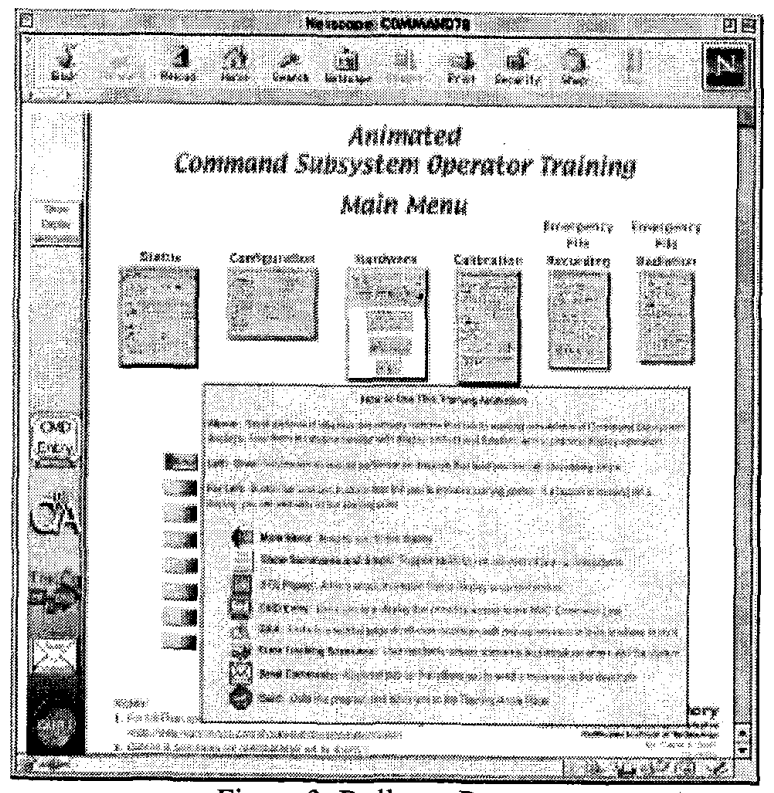

Figure 3: Rollover Pop-up 


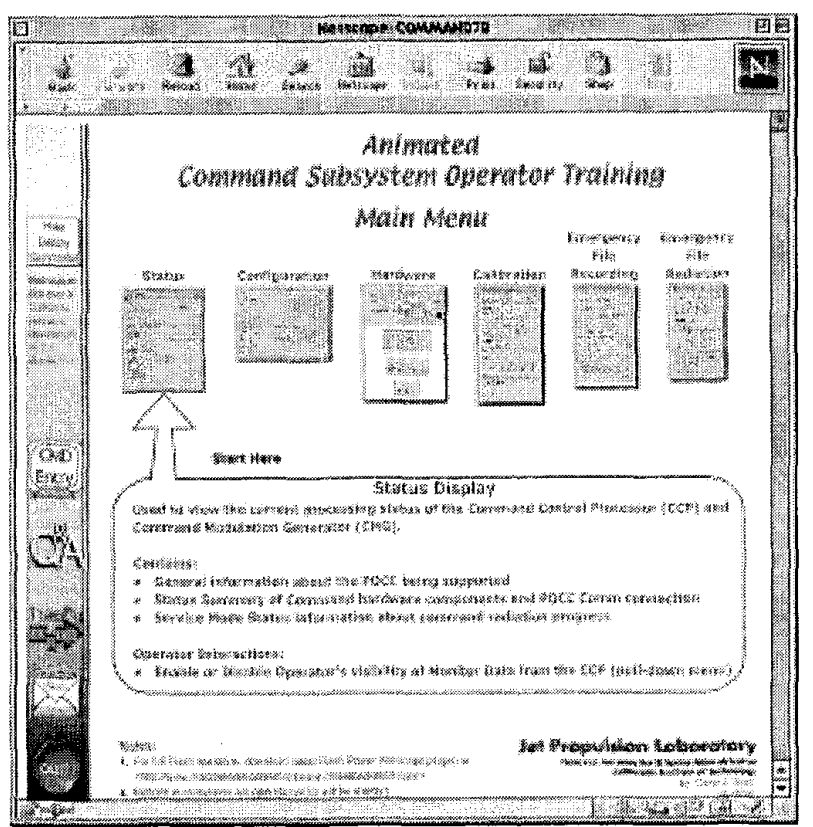

Figure 4: Display Summary Pop-up

To begin the training, the operator clicks the second green button in the Main Menu (seen in Figure 2) to display the startup environment (Figure 5.) The actual workstation uses four monitors to support an assortment of displays from multiple subsystems. To make the display readable by the operator, the main connection display will expand to full size when the operator clicks its button in the left monitor.

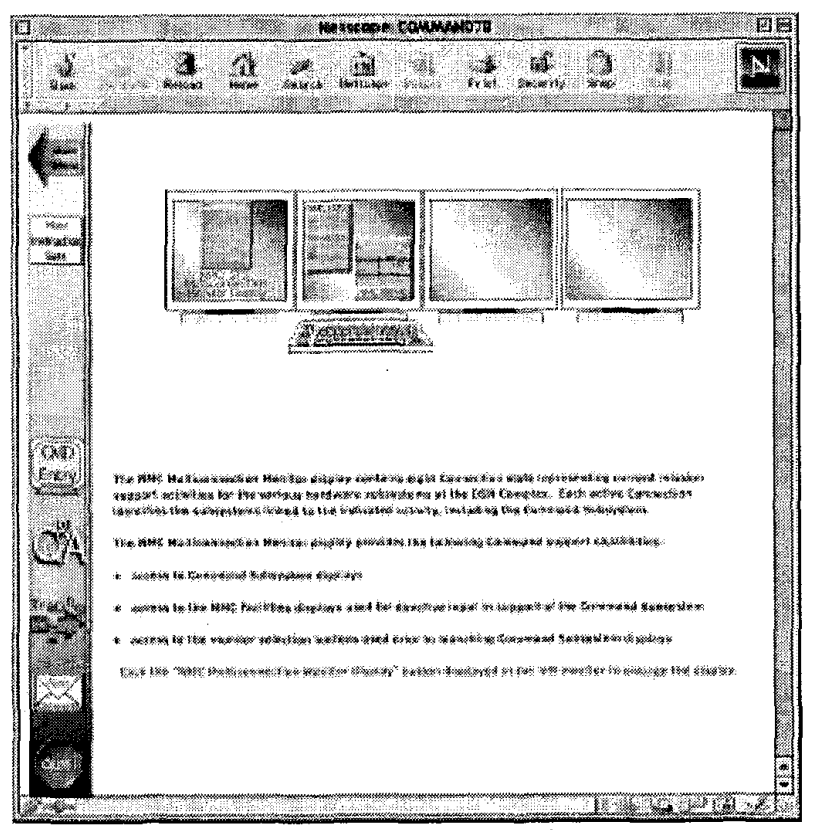

Figure 5: The Workstation Environment

The full-sized GUI appears with instructions for bringing up the Command Subsystem menu. Most users begin with the
Status display (Figure 6) for monitoring commanding activities during the track; it is left to the learner to decide the sequence of the training.

The original animation constrained the operator to a sequential learning flow. The delivered version assumed a more sophisticated user familiar with station hardware and interfaces. For software simulation training of this type, the information presented must be clear and comprehensive, subtle in nature, allowing the basics to be available when needed and not obstructing the more advanced nature of system operation. By enabling the student to select the course of action, the student maneuvers immediately to the point of interest without having to suffer through layers of already familiar material.

Changes to individual GUI displays were incorporated to make them operate exactly as the real subsystem. Initial attempts at building menus treated individual options as separate clunky buttons overlaying the rectangular menu background. Exploring more precise duplication methods resulted in near facsimiles of subsystem menus and buttons.

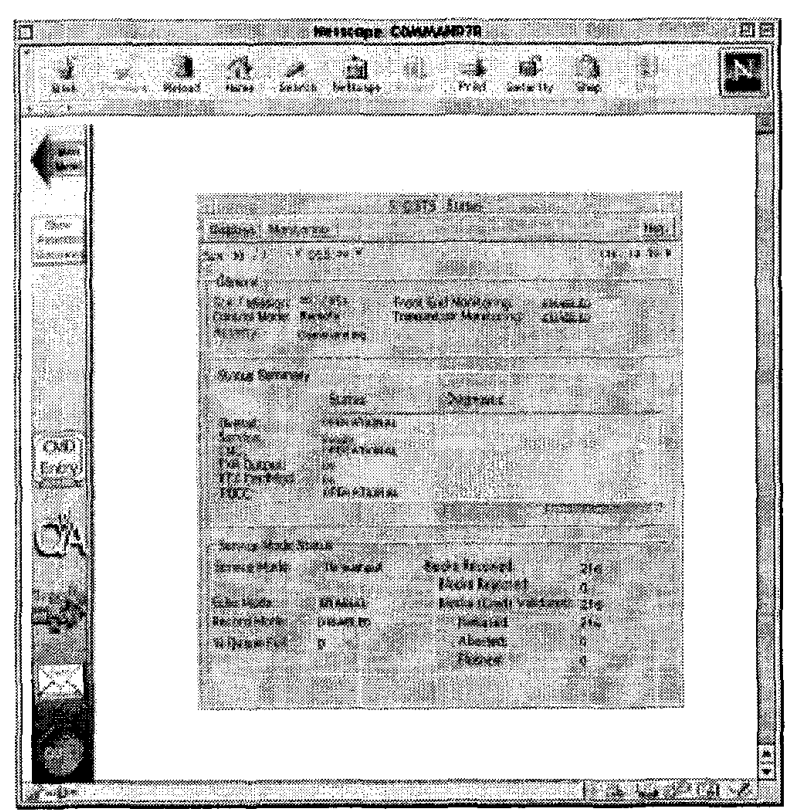

Figure 6: Status Display

Buttons on the displays produce realistic results. For instance, the operator simulates the disabling of the Transmitter monitoring just as it is performed on the real subsystem (see Figure 7.) In addition, the values update to simulate that the Project Operations Control Center (POCC) is radiating commands (lower right column of values in Figure 7.) The POCC is controlling radiation remotely while the Command Subsystem is monitoring their activities. 


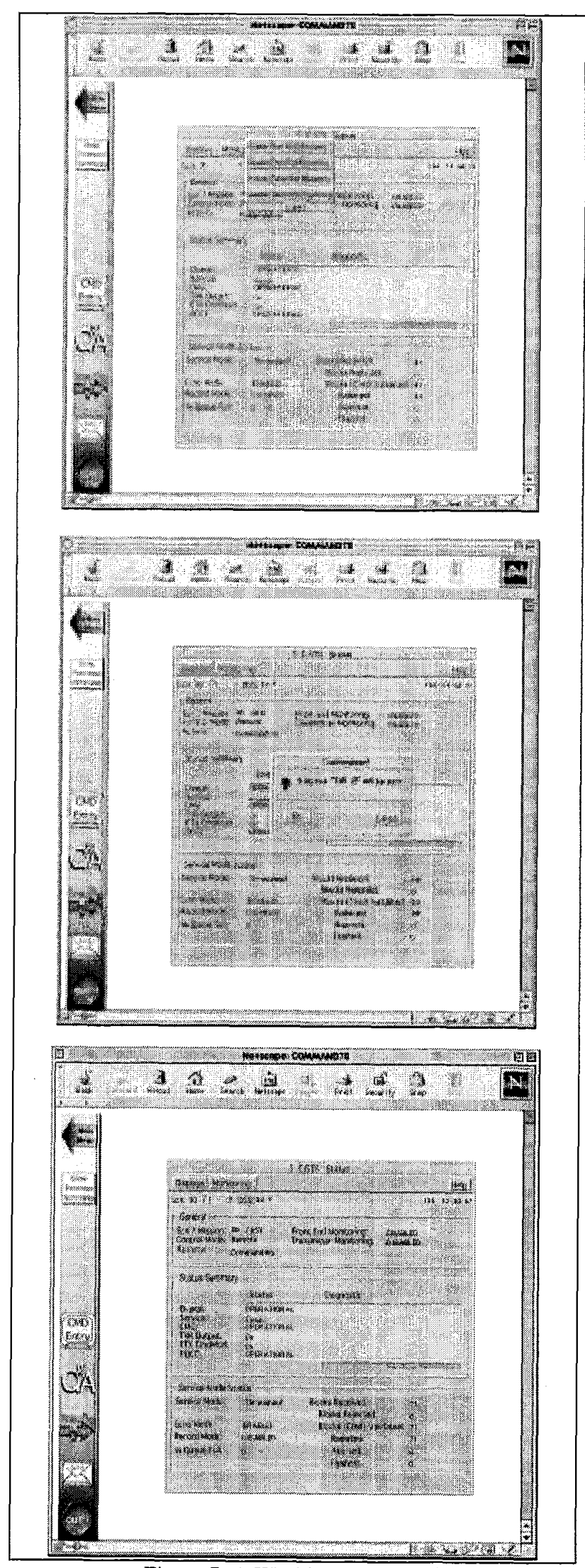

Figure 7: A Working Menu Option

\section{Changes In Code Structure}

Objects that performed action (movement or data results) were better managed as movie clips. Movie clips appear invisibly for call or recall to immediate action. A justification for using movie clips is that the main timeline is less populated, making it easier to manipulate. The use of movie clips limits the timeline to fewer frames. Each movie clip contains its own unique timeline. Our original calibration time line was several hundred frames and nearly totally sequential and inflexible. When converted, the main timeline called three movie clips, and the movie clips, in turn, called several additional movie clips. Consequently, the Calibration display's timeline was 80 frames, and of those, many were blanks employed for readability.

Layering was an exercise in patience. It was easy to add objects to the time line, however, if any object needed attention (editing, moving, locating, etc.) it was not easy to isolate and manipulate unless it was on a layer of its own. Therefore, objects (like buttons, images, or variables) or object groups (like masks, static text, or navigational instructions) were given their own layers. This made development time-consuming, while allowing debugging and maintenance to become nearly effortless. The Calibration display's timeline contained 102 layers (see working display scenario in Figure 8.)

Figure 8 describes: a) the initial calibration display with instructions to, b) set a spacecraft value of $0, c$ ) calibrate an internal board, d) calibrate the processing hardware, and e, f) perform signal calibration. A student apprentice (APT) created the spectrum analyzer cartoon. The intent of replicating these displays is to capture the visual changes on the displays based on operator input, not to focus on the textual content of the displays.

For non-programming engineers and trainers, Flash is an excellent choice for creating simulations because animation evolves in such a manner that action choices are presented to the developer as options to be assigned to objects or frames. By carefully sequencing objects in frames, selecting strategic actions, and learning to use other inherent capabilities of the program, a "thousand words" can be easily reduced to an animated picture. 


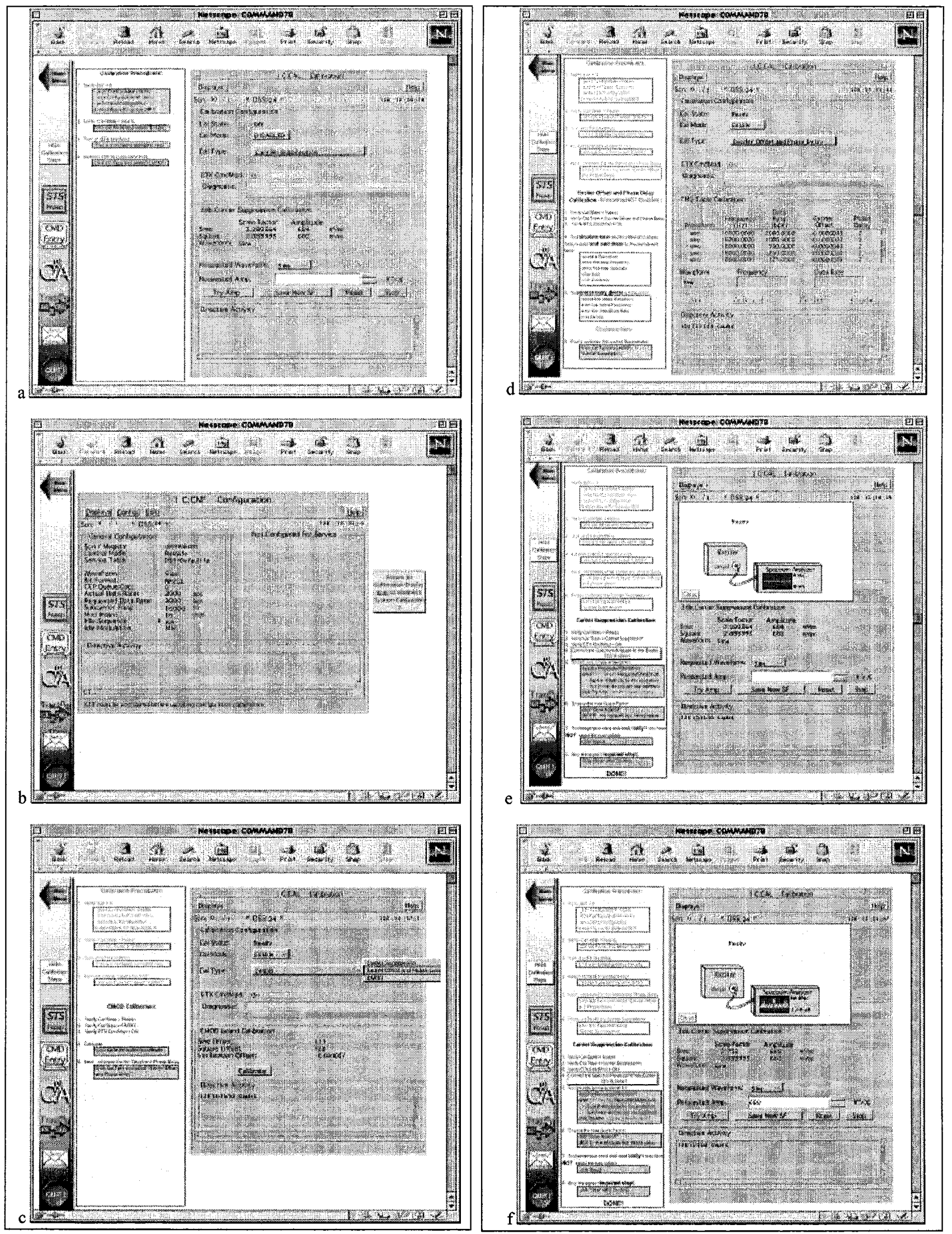

Figure 8: Functionality in Layers and Frames 


\section{ChANGES IN TRAINING SCENARIOS}

On-site subsystem training was planned as a two-phase effort:

1) Initial classroom training - preparation for the deliverable Command Subsystem.

2) Hands-on instruction - using the operational Command Subsystem.

The animated Command simulation was developed as a learning tool to provide access to realistic displays providing familiarity with operation and to gain proficiency in using them.

When subsystem delivery dates were in close proximity, all resources were dedicated to test and integration at the three DSN sites. Station personnel required immediate training, and remote training via video conferencing was considered. A training process, to be successful, requires that the trainee gets to "do it", rather than watch it. Previous remote handson software training experiences had been partially successful, although painfully time-consuming with screen visibility fuzzy and audience actions not being visible by the instructor.

A decision to use Microsoft's NetMeeting with a separate voice line for communication and verbal instructions was made. A NetMeeting session was configured to enable an Internet browser to run the animated simulation on a host machine at JPL, while three machines at Canberra, Australia, each logged into the JPL host machine. Fourteen DSN operators participated at the meeting huddled around the three machines. The participants at Canberra took physical control of the program running on JPL's host machine. A separate voice line used speakerphones to avoid impairment of response time and to enable everyone in attendance to hear and speak. The instructor gave voice instructions and monitored the action as the operators took control of the simulation from their machines via their own mouse and keyboard. The slight screen delay was nearly imperceptible as the training proceeded. The entire session took a little over an hour and the response was extremely positive.

The response was so enthusiastic that all initial training at Canberra and Madrid was performed using the animated simulation via NetMeeting. Additional training on the real subsystem was provided during or prior to user acceptance testing.

\section{DeLIVERy Bonus}

The benefits of a training simulation were apparent prior to subsystem delivery at the DSN's Goldstone Deep Space Communication Complex. With the original delivery timeline, training would coincide with the end of integration testing. The subsystem was performing as expected and it was assumed that training would use the unused portions of scheduled subsystem test time. During the transition from test hardware to operational hardware, the usual unexpected problems were encountered. When the Training team arrived at the Goldstone complex, the workstation containing the new Command delivery was unavailable to trainers, as massive re-testing was the priority of the day.

Subsystem delivery required trained operators. On-site testing required constant usage of the subsystem, providing limited or no opportunity for operators to experience the actual subsystem procedural indoctrination and training. The training simulation, which was developed as a learning and practice module usually delivered via the Internet, was usable in stand-alone mode via the browser (without Internet connection), so it was available as the primary learning resource for trainers. The versatility of web-based animated training for mission operations was proven during this training experience and operator training was declared an on-time delivery item.

\section{CONCLUSIONS}

Lessons learned from the training development effort incorporate the resolution of problems in module design and structure, simplification of methods, version control, customer input, object control and orientation, planning, scheduling... the same design and development issues of a typical software development effort, with an entirely different focus. The Training focus is on the customer, making the operators' job easier, or at least, easier to learn. With that in mind, and by developing training concurrent with system development, there was a cross-pollination of developer-trainer-operator feedback. That customer focus is the key element to on-line training development.

\section{REFERENCE}

[1] Carol J. Scott, "Vector Animation: Web-Based Software Training On Demand," 2000 IEEE Aerospace Conference Proceedings, March 18-25, 2000.

[2] Carol J. Scott, "Web-Based Software Simulations: Training Tools With a Diangostic Flavor," Fourth IAA International Conference Proceedings on Low-Cost Planetary Missions, May 2-5, 2000.

\section{ACKNOWLEDGEMENT}

The Jet Propulsion Laboratory, California Institute of Technology, under a contract with the National Aeronautics and Space Administration is carrying out the research described in this paper. 


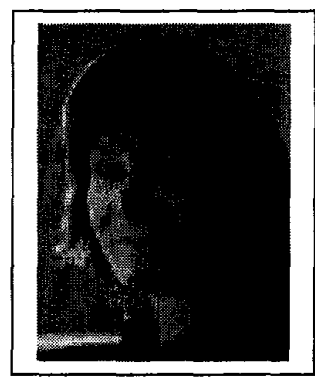

Carol Scott-- is a Lead Information Technology Specialist at the Jet Propulsion Laboratory where she has designed and developed software training for mission flight teams and DSN operators for the past ten years. She has a BS in Computer Science from Chapman College and $a B A$ in English from the University of Nebraska at Omaha. She has authored five papers on training development. 